\title{
Dipeptidyl peptidase-4 inhibitors and incidence of inflammatory bowel disease among patients with type 2 diabetes: population based cohort study
}

\author{
Devin Abrahami, ${ }^{1,2}$ Antonios Douros, ${ }^{1,2,3}$ Hui Yin, ${ }^{1}$ Oriana Hoi Yun Yu, ${ }^{1,4}$ Christel Renoux, ${ }^{1,2,5}$ \\ Alain Bitton, ${ }^{6,7}$ Laurent Azoulay ${ }^{1,2,8}$
}

\begin{abstract}
${ }^{1}$ Centre for Clinical
Epidemiology, Lady Davis

Institute, Jewish General

Hospital, Montreal, QC, Canada

H3T 1 E2

${ }^{2}$ Department of Epidemiology, Biostatistics, and Occupational Health, McGill University,

Montreal, QC, Canada

${ }^{3}$ Institute of Clinical

Pharmacology and Toxicology,

Charité - Universitätsmedizin

Berlin, Berlin, Germany

${ }^{4}$ Division of Endocrinology,

Jewish General Hospital,

Montreal, QC, Canada
\end{abstract}

${ }^{5}$ Department of Neurology and Neurosurgery, McGill University,

Montreal, QC, Canada

${ }^{6}$ Division of Gastroenterology,

Department of Medicine,

McGill University, Montreal, QC,

Canada

${ }^{7}$ McGill University Health

Centre, Montreal, QC, Canada

${ }^{8}$ Gerald Bronfman Department of Oncology, McGill University, Montreal, QC, Canada

Correspondence to: L Azoulay laurent.azoulay@mcgill.ca

Additional material is published online only. To view please visit the journal online.

Cite this as: $B M J$ 2018;360:k872 http://dx.doi.org/10.1136/bmj.k872

Accepted: 13 February 2018

\section{ABSTRACT}

OBJECTIVE

To assess whether the use of dipeptidyl peptidase- 4 inhibitors is associated with the incidence of inflammatory bowel disease in patients with type 2 diabetes.

\section{DESIGN}

Population based cohort study.

\section{SETTING}

More than 700 general practices contributing data to the United Kingdom Clinical Practice Research Datalink.

\section{PARTICIPANTS}

A cohort of 141170 patients, at least 18 years of age, starting antidiabetic drugs between 1 January 2007 and 31 December 2016, with follow-up until 30 June 2017.

\section{MAIN OUTCOME MEASURES}

Adjusted hazard ratios for incident inflammatory bowel disease associated with use of dipeptidyl peptidase-4 inhibitors overall, by cumulative duration of use, and by time since initiation, estimated using time dependent Cox proportional hazards models. Use of dipeptidyl peptidase-4 inhibitors was modelled as a time varying variable and compared with use of other antidiabetic drugs, with exposures lagged by six months to account for latency and diagnostic delays.

RESULTS

During 552413 person years of follow-up, 208 incident inflammatory bowel disease events occurred (crude incidence rate of 37.7 (95\% confidence interval 32.7 to 43.1) per 100000 person years). Overall, use of dipeptidyl peptidase-4 inhibitors was associated

\section{WHAT IS ALREADY KNOWN ON THIS TOPIC}

The effect of the dipeptidyl peptidase-4 (DPP-4) enzyme in autoimmune diseases such as inflammatory bowel disease (IBD) is not well understood

Low concentrations of the DPP- 4 enzyme have been associated with increased IBD activity, although the direction of this association remains unclear

No observational studies have investigated the association between the use of DPP-4 inhibitors and the incidence of IBD

\section{WHAT THIS STUDY ADDS}

Use of DPP-4 inhibitors was associated with an overall $75 \%$ increase in the risk of IBD

This association was elevated between three and four years of use and between two and four years after the start of treatment

These findings need to be replicated, but physicians should be made aware of this possible association

with an increased risk of inflammatory bowel disease (53.4 $v 34.5$ per 100000 person years; hazard ratio $1.75,95 \%$ confidence interval 1.22 to 2.49 ). Hazard ratios gradually increased with longer durations of use, reaching a peak after three to four years of use (hazard ratio $2.90,1.31$ to 6.41 ) and decreasing after more than four years of use $(1.45,0.44$ to 4.76$)$. A similar pattern was observed with time since starting dipeptidyl peptidase-4 inhibitors. These findings remained consistent in several sensitivity analyses.

CONCLUSIONS

In this first population based study, the use of dipeptidyl peptidase- 4 inhibitors was associated with an increased risk of inflammatory bowel disease. Although these findings need to be replicated, physicians should be aware of this possible association.

\section{Introduction}

The use of dipeptidyl peptidase- 4 inhibitors in the treatment of type 2 diabetes has increased considerably since their introduction a decade ago. ${ }^{1}$ These second to third line treatments have been shown to have favourable effects compared with other antidiabetic drugs, such as lowering the risk of hypoglycaemia and having neutral effects on body weight and cardiovascular outcomes. ${ }^{2-4}$ These effects are mediated by inhibition of the dipeptidyl peptidase-4 enzyme leading to a rise in glucagon-like peptide 1 concentrations, ${ }^{2}$ but inhibition may also have unintended effects. The dipeptidyl peptidase- 4 enzyme is found in the serum and has been associated with several different cellular functions. ${ }^{5}$ It is also expressed on the surface of a variety of cell types, including those involved in immune response. ${ }^{67}$

The effect of the dipeptidyl peptidase- 4 enzyme in autoimmune conditions such as inflammatory bowel disease is not well understood. On the one hand, studies in mouse models of inflammatory bowel disease suggest that treatment with dipeptidyl peptidase-4 inhibitors results in decreased disease activity. ${ }^{7-10}$ On the other hand, clinical data indicate that patients with inflammatory bowel disease have lower serum dipeptidyl peptidase-4 enzyme concentrations than healthy controls. ${ }^{6} 1112$ Moreover, such lower concentrations are inversely associated with increased disease activity, although whether this is the cause or consequence of active disease is unclear. $^{12}{ }^{13}$ To date, the association between dipeptidyl peptidase- 4 enzyme concentrations and incident inflammatory bowel disease has not been studied. 
To our knowledge, no observational study has specifically investigated the association between use of dipeptidyl peptidase- 4 inhibitors and the incidence of inflammatory bowel disease. Thus, the objective of this population based study was to determine whether the use of dipeptidyl peptidase-4 inhibitors is associated with the incidence of inflammatory bowel disease in patients with type 2 diabetes.

\section{Methods}

Data source

This study used data from the Clinical Practice Research Datalink (CPRD), a primary care database from the UK. The CPRD records demographic and lifestyle information, prescription data, referrals, and diagnoses for more than 15 million patients in more than 700 general practices. These data are representative of the general UK population and have been shown to be of high quality and validity. ${ }^{14-16}$ The CPRD uses the Read code classification for medical diagnoses and procedures, ${ }^{17}$ and a coded drug dictionary based on the British National Formulary for prescription details.

\section{Study population}

We identified a base cohort of patients, at least 18 years of age, newly treated with non-insulin antidiabetic drugs (metformin, sulfonylureas, meglitinides, thiazolidinediones, acarbose, dipeptidyl peptidase-4 inhibitors, glucagon-like peptide 1 receptor agonists, and sodium-glucose co-transporter-2 inhibitors) between 1 January 1988 and 31 December 2016. Patients were required to have at least one year of medical history in the CPRD before their initial prescription. We excluded patients treated with insulin at any time before their initial prescription for a non-insulin antidiabetic drug (that is, patients with advanced disease) and female patients with a history of polycystic ovary syndrome (at any time before their initial prescription) or a history of gestational diabetes (in the year before their initial prescription), as these are other indications for metformin.

Within the base cohort, we assembled a study cohort of patients who started a new antidiabetic drug class not previously used in their treatment history in or after 2007 (the year the first dipeptidyl peptidase-4 inhibitor, sitagliptin, entered the UK market). ${ }^{3}$ This cohort thus included patients newly treated for diabetes, as well as those for whom treatment was newly modified (addons or switches). Cohort entry was the date of this new antidiabetic prescription. At this stage, we excluded patients previously diagnosed as having inflammatory bowel disease, including those previously exposed to mesalamine, at any time before cohort entry (Crohn's disease and ulcerative colitis; Read codes listed in supplementary table A). Diagnoses of inflammatory bowel disease have been previously validated in the CPRD, with positive predictive values above $90 \% .{ }^{18-20}$ We also excluded patients with a history of diverticulitis, ischaemic colitis, pseudomembranous colitis, or unspecific colitis (common differential diagnoses for inflammatory bowel disease ${ }^{21}$ ) at any time before cohort entry. Finally, we excluded patients with less than six months of follow-up after cohort entry to account for a latency period and known diagnostic delays of inflammatory bowel disease. ${ }^{22}$ All patients were followed starting six months after cohort entry until an incident diagnosis of inflammatory bowel disease or censored on an incident diagnosis of ischaemic colitis or diverticulitis, death from any cause, end of registration with the general practice, or the end of the study period (30 June 2017), whichever occurred first.

\section{Exposure assessment}

We modelled the use of dipeptidyl peptidase-4 inhibitors (alone or in combination with other antidiabetic drugs) as a time varying variable and compared it with the use of all other antidiabetic drugs. As part of this exposure definition, patients could move from a period of non-exposure to a period of exposure after a six month lag period (allowing them to contribute both unexposed and exposed person time). Thus, patients were considered exposed starting six months after their first prescription until the end of the follow-up period, analogous to an intention to treat approach. Consequently, we considered inflammatory bowel disease events occurring during the six month lag period to be unexposed events. The use of a lag period was necessary for latency considerations, given that exposures of short duration are unlikely to be associated with the incidence of inflammatory bowel disease, to account for possible diagnostic delays associated with inflammatory bowel disease, ${ }^{22}$ and to reduce detection bias and reverse causality. Finally, we deemed the comparator group of other antidiabetic drugs to be appropriate, as none of these drugs has been previously associated with the incidence of inflammatory bowel disease. We considered this to be the definition of primary exposure.

We also considered two definitions of secondary exposure. The first assessed the association according to cumulative duration of dipeptidyl peptidase- 4 inhibitor use. We defined this time dependent variable by summing the durations associated with each prescription up until time of event. The second assessed time since initiation, which we defined in a time dependent fashion as the time between the first dipeptidyl peptidase- 4 inhibitor prescription and time of event.

\section{Potential confounders}

The models were adjusted for the following potential confounders measured at cohort entry: age, sex, year of cohort entry, body mass index, alcohol related disorders (alcoholism, alcoholic cirrhosis of the liver, alcoholic hepatitis, and hepatic failure), and smoking status. We also adjusted for haemoglobin $A_{1 c}$ (last laboratory result before cohort entry), microvascular (nephropathy, neuropathy, retinopathy) and macrovascular (myocardial infarction, stroke, peripheral arteriopathy) complications of diabetes 
(at any time before cohort entry), duration of treated diabetes, and antidiabetic drugs used before cohort entry, as proxies for disease severity. Age and duration of treated diabetes were modelled flexibly as continuous variables by using cubic spline models to account for possible non-linear relations with the outcome. The models also considered the use of aspirin, non-steroidal anti-inflammatory drugs, hormonal replacement therapy, oral contraceptives, other autoimmune conditions (all at any time before cohort entry), ${ }^{23}$ as well as the total number of unique non-antidiabetic drugs received in the year before cohort entry as a general measure of comorbidity. ${ }^{24}$

\section{Statistical analysis}

We calculated crude incidence rates of inflammatory bowel disease with 95\% confidence intervals based on the Poisson distribution for the entire cohort and for each exposure group. For all analyses, we used time dependent Cox proportional hazards models to estimate hazard ratios and 95\% confidence intervals for inflammatory bowel disease associated with the use of dipeptidyl peptidase-4 inhibitors compared with the use of other antidiabetic drugs. The models were adjusted for the potential confounders listed above. We also calculated the number needed to harm for patients followed over a two year and four year period by using methods accounting for varying patient follow-up times. ${ }^{25}$

\section{Secondary analyses}

We did four secondary analyses. Firstly, we assessed whether a duration-response relation existed according to cumulative duration of use by estimating hazard ratios for five predefined duration categories ( $\leq 1$ year, 1.1-2 years, 2.1-3 years, 3.1-4 years, and $>4$ years). Secondly, we investigated the association with time since initiation by estimating hazard ratios for three predefined categories ( $\leq 2$ years, 2.1-4 years, and $>4$ years). We also modelled cumulative duration of use and time since initiation as continuous variables by using restricted cubic splines. Thirdly, to investigate the possibility of a drug specific effect, we repeated the analysis stratifying by type of dipeptidyl peptidase-4 inhibitor (sitagliptin, saxagliptin, and other). Finally, we repeated the primary analysis by stratifying on type of inflammatory bowel disease (Crohn's disease, ulcerative colitis, and unspecified disease).

\section{Sensitivity analyses}

We did 11 sensitivity analyses to assess the robustness of our findings. Firstly, given uncertainties related to the length of the lag period, we increased the exposure lag period to one year. Secondly, to assess the validity of our outcome definition, we restricted inflammatory bowel disease events to those accompanied by clinically relevant supporting events (supplementary methods 1). Thirdly, to investigate the effect of informative censoring, we did a competing risk analysis by death from any cause, using the Fine and
Gray subdistribution model. ${ }^{26}$ Fourthly, to investigate the effect of detection bias from undiagnosed inflammatory bowel disease, we stratified the cohort by age at cohort entry ( $<60$ and $\geq 60$ years). In the UK, patients aged 60-74 years are invited for faecal occult blood tests every two years as part of the Bowel Cancer Screening Programme. ${ }^{27}$ Fifthly, we used a stricter exposure definition, in which dipeptidyl peptidase-4 inhibitor use was redefined as receipt of at least four prescriptions within a 12 month moving window; we considered patients to be exposed only six months after the fourth qualifying prescription. Sixthly, to account for a possible incretin effect of glucagon-like peptide 1 receptor agonists, we redefined exposure into four mutually exclusive categories: dipeptidyl peptidase-4 inhibitors (alone or in combination, excluding glucagon-like peptide 1 receptor agonists), glucagon-like peptide 1 receptor agonists (alone or in combination, excluding dipeptidyl peptidase-4 inhibitors), both dipeptidyl peptidase- 4 inhibitor and glucagon-like peptide 1 receptor agonists, and other antidiabetic drugs (new reference category). Seventhly, to assess the possibility of anti-inflammatory effects of thiazolidinediones, ${ }^{28}$ we excluded patients treated with thiazolidinediones at any time before cohort entry and censored them on initiation during follow-up. The eighth to tenth analyses assessed the effect of residual confounding by conducting a marginal structural model (using inverse probability of treatment and censoring weighting), disease risk score, and multiple imputation for variables with missing information (supplementary methods 2-4). Finally, in a post hoc sensitivity analysis, we used the rule out method to estimate the strength of an unknown or unmeasured confounder that would be needed to move the observed hazard ratio to the null. ${ }^{29}$

\section{Ancillary analyses}

We did two ancillary analyses to further assess the validity of our findings. The first used insulin as a negative control exposure, ${ }^{30}$ a last line treatment that has not been associated with inflammatory bowel disease. For this analysis, we excluded prevalent users of insulin before cohort entry and modelled new use of insulin as a time dependent variable lagged by six months. The second was a head to head comparison of patients newly treated with dipeptidyl peptidase- 4 inhibitors versus insulin between 1 January 2007 and 31 December 2016, with follow-up until 30 June 2017. For this analysis, a Cox proportional hazard model was stratified on fifths of propensity score (supplementary methods 5). We used SAS version 9.4 for all the analyses described above.

\section{Patient involvement}

We did not include patients as study participants, as our study involved the use of secondary data. Patients were not involved in the design or implementation of the study. We do not plan to involve patients in the dissemination of results, nor will we disseminate results directly to patients. 


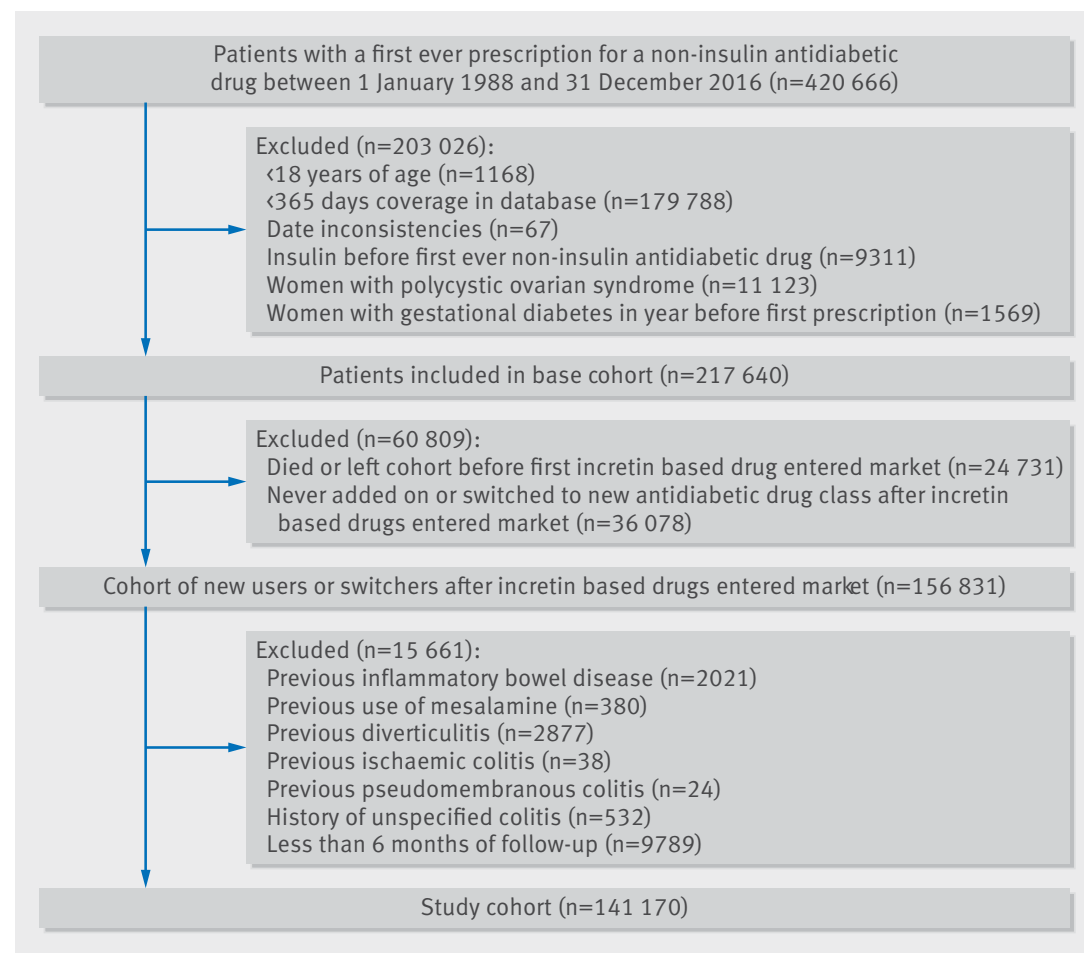

Fig 1 | Flowchart of patients included in base and study cohorts

\section{Results}

We included 141170 patients in the cohort (fig 1). These patients were followed for a median of 3.6 (interquartile range 1.6-5.9) years beyond the six month post-cohort entry lag period. During 552413 person years of follow-up, 208 incident inflammatory bowel disease events occurred, generating an incidence rate of 37.7 (95\% confidence interval 32.7 to 43.1) per 100000 person years. Nearly all these events $(n=193 ; 92.8 \%)$ had at least one clinically relevant supporting event (supplementary table B). Overall, 30488 $(21.6 \%)$ patients received at least one prescription for a dipeptidyl peptidase- 4 inhibitor during the study period; the median duration of use was 1.6 (interquartile range 0.7-3.1) years.

Table 1 shows the baseline characteristics of the entire cohort and the cohort stratified by drug use at cohort entry. Compared with users of other antidiabetic drugs, dipeptidyl peptidase-4 inhibitor users were older, more likely to have higher haemoglobin $\mathrm{A}_{1 \mathrm{c}}$ concentrations, more likely to have a longer duration of treated diabetes, and more likely to have microvascular complications of diabetes. Users of dipeptidyl peptidase-4 inhibitors were also more likely to have used aspirin and non-steroidal anti-inflammatory drugs but less likely to have used oral contraceptives.

Table 2 shows the results of the primary and secondary analyses. Compared with use of other antidiabetic drugs, use of dipeptidyl peptidase-4 inhibitors was associated with a $75 \%$ increase in risk of inflammatory bowel disease $(53.4 v 34.5$ per
100000 per year; hazard ratio $1.75,95 \%$ confidence interval 1.22 to 2.49). The number needed to harm corresponded to 2291 patients followed over a two year period and 1177 over a four year period. In secondary analyses, hazard ratios gradually increased with longer durations of use, reaching a peak after three to four years of use (hazard ratio 2.90, 1.31 to 6.41) and decreasing after more than four years of use (1.45, 0.44 to 4.76). A similar pattern was observed with time since initiation, with the highest hazard ratio observed between two and four years after initiation (2.50, 1.57 to 3.99) and a decrease after more than four years $(1.75,0.86$ to 3.58$)$. These patterns remained consistent in the cubic spine models (supplementary figures $\mathrm{A}$ and $\mathrm{B}$ ).

Overall, no single dipeptidyl peptidase-4 inhibitor drug was statistically associated with inflammatory bowel disease, although the strata had few events (supplementary table C). In analyses stratified on type of inflammatory bowel disease, the use of dipeptidyl peptidase- 4 inhibitors was associated with a greater than twofold increase in risk of ulcerative colitis (hazard ratio 2.23, 1.32 to 3.76 ), whereas no statistically significant association was observed with Crohn's disease $(0.87,0.37$ to 2.09$)$ (supplementary table D).

\section{Sensitivity and ancillary analyses}

Figure 2 summarises the results of the sensitivity analyses (shown in detail in supplementary tables E-N and supplementary figure C). Overall, these analyses produced results that were consistent with those of the primary analysis, with statistically significant hazard ratios ranging between 1.60 and 2.21. The negative control analysis comparing the use of insulin with the use of other antidiabetic drugs yielded a hazard ratio close to the null value $(0.92,0.53$ to 1.58 ; table $3)$. In the head to head comparison, use of dipeptidyl peptidase- 4 inhibitors was associated with a greater than twofold increase in risk of inflammatory bowel disease, compared with insulin (hazard ratio 2.28, 1.07 to 4.85 ) (table 3, supplementary figure D and supplementary table 0 ).

\section{Discussion}

To our knowledge, this is the first observational study to specifically investigate the association between the use of dipeptidyl peptidase-4 inhibitors and the incidence of inflammatory bowel disease. Use of dipeptidyl peptidase-4 inhibitors was associated with an overall $75 \%$ increase in risk of inflammatory bowel disease. In secondary analyses, the association was particularly elevated between three and four years of use and between two and four years after the start of dipeptidyl peptidase- 4 inhibitor treatment. This gradual increase in the risk is consistent with the hypothesis of a possible delayed effect of the use of dipeptidyl peptidase- 4 inhibitors on the incidence of inflammatory bowel disease. This association remained highly consistent across a variety of sensitivity analyses. 

Table 1 | Baseline characteristics of entire cohort and cohort stratified by drug use at
cohort entry. Values are numbers (percentages) unless stated otherwise

\begin{tabular}{|c|c|c|c|}
\hline \multirow[b]{2}{*}{ Characteristic } & \multirow[b]{2}{*}{$\begin{array}{l}\text { Entire cohort } \\
(n=141170)\end{array}$} & \multicolumn{2}{|c|}{ Use at cohort entry } \\
\hline & & $\begin{array}{l}\text { DPP-4 inhibitors } \\
(n=7231)\end{array}$ & $\begin{array}{l}\text { Other antidiabetic } \\
\text { drugs }(n=133939)\end{array}$ \\
\hline Mean (SD) age, years & $61.6(13.6)$ & $66.1(11.8)$ & $61.4(13.6)$ \\
\hline Male sex & 80995 (57.4) & $4164(57.6)$ & 76831 (57.4) \\
\hline \multicolumn{4}{|l|}{ Year of cohort entry: } \\
\hline 2007 & $20368(14.4)$ & $126(1.7)$ & $20242(15.1)$ \\
\hline 2008 & $18658(13.2)$ & $506(7.0)$ & $18152(13.6)$ \\
\hline 2009 & $18801(13.3)$ & $1014(14.0)$ & $17787(13.3)$ \\
\hline 2010 & $17507(12.4)$ & $1485(20.5)$ & $16022(12.0)$ \\
\hline 2011 & $14701(10.4)$ & $1103(15.3)$ & $13598(10.2)$ \\
\hline 2012 & $13788(9.8)$ & $983(13.6)$ & $12805(9.6)$ \\
\hline 2013 & $12214(8.7)$ & $744(10.3)$ & $11470(8.6)$ \\
\hline 2014 & $9896(7.0)$ & $544(7.5)$ & $9352(7.0)$ \\
\hline 2015 & $8731(6.2)$ & $451(6.2)$ & $8280(6.2)$ \\
\hline 2016 & $6506(4.6)$ & $275(3.8)$ & $6231(4.7)$ \\
\hline \multicolumn{4}{|l|}{ Body mass index: } \\
\hline$<25$ & $14743(10.4)$ & $736(10.2)$ & $14007(10.5)$ \\
\hline $25-30$ & $41434(29.4)$ & $2127(29.4)$ & 39307 (29.3) \\
\hline$\geq 30$ & $81993(58.1)$ & $4337(60.0)$ & $77656(58.0)$ \\
\hline Unknown & $3000(2.1)$ & $31(0.4)$ & $2969(2.2)$ \\
\hline Alcohol related disorders & $20782(14.7)$ & $1431(19.8)$ & $19351(14.5)$ \\
\hline \multicolumn{4}{|l|}{ Smoking status: } \\
\hline Current smoker & $22812(16.2)$ & $940(13.0)$ & $21872(16.3)$ \\
\hline Past smoker & $51490(36.5)$ & $2817(39.0)$ & $48673(36.3)$ \\
\hline Never smoker & $66350(47.0)$ & $3467(47.9)$ & $62883(47.0)$ \\
\hline Unknown & $518(0.4)$ & $7(0.1)$ & $511(0.4)$ \\
\hline \multicolumn{4}{|l|}{ Haemoglobin $\mathrm{A}_{1}$ : } \\
\hline$\leq 7.0 \%$ & 25508 (18.1) & $1325(18.3)$ & $24183(18.1)$ \\
\hline $7.1-8.0 \%$ & $30720(21.8)$ & $2424(33.5)$ & $28296(21.1)$ \\
\hline$>8.0 \%$ & $43227(30.6)$ & $3170(43.8)$ & $40057(29.9)$ \\
\hline Unknown & $41715(29.6)$ & $312(4.3)$ & 41403 (30.9) \\
\hline Nephropathy & $34573(24.5)$ & $2855(39.5)$ & $31718(23.7)$ \\
\hline Neuropathy & $14564(10.3)$ & $1838(25.4)$ & $12726(9.5)$ \\
\hline Retinopathy & $15249(10.8)$ & $2348(32.5)$ & $12901(9.6)$ \\
\hline Myocardial infarction & $9627(6.8)$ & $598(8.3)$ & $9029(6.7)$ \\
\hline Stroke & $6844(4.9)$ & $463(6.4)$ & $6381(4.8)$ \\
\hline Peripheral arteriopathy & $5010(3.6)$ & $417(5.8)$ & $4593(3.4)$ \\
\hline Mean (SD) duration of treated & $1.3(3.0)$ & $7.7(4.2)$ & $1.0(2.5)$ \\
\hline
\end{tabular}

diabetes, years

Class of antidiabetic drugs*:

\begin{tabular}{llll}
\hline Metformin & $27265(19.3)$ & $6482(89.6)$ & $20783(15.5)$ \\
\hline Sulfonylureas & $13522(9.6)$ & $4032(55.8)$ & $9490(7.1)$ \\
\hline Thiazolidinediones & $7154(5.1)$ & $2462(34.1)$ & $4692(3.5)$ \\
\hline Insulin & $1213(0.9)$ & $373(5.2)$ & $840(0.6)$ \\
\hline Other & $939(0.7)$ & $277(3.8)$ & $662(0.5)$ \\
\hline Aspirin & $60329(42.7)$ & $4760(65.8)$ & $55569(41.5)$ \\
\hline $\begin{array}{l}\text { Non-steroidal anti-inflammatory } \\
\text { drugs }\end{array}$ & $76366(54.1)$ & $4600(63.6)$ & $71766(53.6)$ \\
\hline Hormonal replacement therapy & $17202(12.2)$ & $1069(14.8)$ & $16133(12.1)$ \\
\hline Oral contraceptives & $11567(8.2)$ & $398(5.5)$ & $11169(8.3)$ \\
\hline Other autoimmune conditions: & $4418(3.1)$ & $250(3.5)$ & $4168(3.1)$ \\
\hline Psoriasis & $1821(1.3)$ & $92(1.3)$ & $1729(1.3)$ \\
\hline Systemic vasculitis & $513(0.4)$ & $18(0.3)$ & $495(0.4)$ \\
\hline Rheumatoid arthritis & $1953(1.4)$ & $123(1.7)$ & $1830(1.4)$ \\
\hline Sjögren's syndrome & $161(0.1)$ & $14(0.2)$ & $147(0.1)$ \\
\hline Systemic lupus erythematosus & $166(0.1)$ & $11(0.2)$ & $155(0.1)$ \\
\hline No of non-antidiabetic drugs: & & & \\
\hline Mean (SD) & $8.2(6.1)$ & $10.5(6.3)$ & $8.0(6.0)$ \\
\hline 0 & $6206(4.4)$ & $59(0.8)$ & $6147(4.6)$ \\
\hline 1 & $7228(5.1)$ & $103(1.4)$ & $7125(5.3)$ \\
\hline 2 & $8839(6.3)$ & $171(2.4)$ & $8668(6.5)$ \\
\hline 3 & $10003(7.1)$ & $254(3.5)$ & $9749(7.3)$ \\
\hline$\geq 4$ & $108894(77.1)$ & $6644(91.9)$ & $102250(76.3)$ \\
\hline DP & & &
\end{tabular}

DPP-4=dipeptidyl peptidase- 4 .

${ }^{*}$ Non-mutually exclusive groups measured at any time before (not including) cohort entry.

\section{Comparison with previous studies}

The dipeptidyl peptidase-4 enzyme is involved in inflammatory response and is known to modulate gastric hormones; these have been shown to be elevated in patients with inflammatory bowel disease. ${ }^{31}$ However, further study of the exact effect of this enzyme in inflammatory bowel disease is needed. Inhibition of this enzyme with dipeptidyl peptidase-4 inhibitors has been shown to reduce disease activity in Crohn's disease by increasing concentrations of glucagon-like peptide 2, an incretin hormone with intestinotrophic effects. ${ }^{7}$ Furthermore, in experimental mouse models of colitis, treatment with dipeptidyl peptidase- 4 inhibitors decreased both disease activity and disease severity, through inhibition of $\mathrm{T}$ cell proliferation and cytokine production and restoration of gut mucosal damage, respectively. ${ }^{8-10}$ However, the available clinical evidence shows a complex relation between the dipeptidyl peptidase-4 enzyme and inflammatory bowel disease activity. Although the expression of dipeptidyl peptidase-4 was elevated on T cells from patients with inflammatory bowel disease, ${ }^{67}$ serum concentrations and activity of dipeptidyl peptidase-4 were lower compared with healthy controls. $^{6} 11 \quad 12$ Moreover, dipeptidyl peptidase-4 enzyme concentrations had an inverse relation with inflammatory bowel disease activity scores, although the direction of this association remains unclear. ${ }^{12} 13$

In contrast to the aforementioned animal studies that have supported a role for dipeptidyl peptidase- 4 inhibitors in the treatment of inflammatory bowel disease, ${ }^{7-10}$ our study focused on incident inflammatory bowel disease, in which dipeptidyl peptidase-4 may have a different biological function. Although one previous observational study reported a decreased risk of a composite of several autoimmune diseases (including inflammatory bowel disease) with the use of dipeptidyl peptidase- 4 inhibitors (hazard ratio $0.68,95 \%$ confidence interval 0.52 to 0.89$),{ }^{28}$ it did not report any findings on inflammatory bowel disease specifically. This decreased risk may have been driven by other diseases included in the composite outcome. Finally, our results indicate that an increased risk with dipeptidyl peptidase- 4 inhibitors may be associated with ulcerative colitis and not Crohn's disease. However, this finding should be interpreted with caution as this stratified analysis was based on few events, generating a wide confidence interval with an upper 95\% confidence limit of 2.09. Thus, our results do not rule out a possible association with Crohn's disease as well. In summary, although our findings need to be replicated, additional studies are also needed to understand the possible mechanism through which dipeptidyl peptidase- 4 inhibitors may increase the risk of inflammatory bowel disease.

\section{Strengths and limitations of study}

This study has several strengths. Firstly, our study design excluded prevalent users, thus eliminating biases associated with their inclusion. ${ }^{32}$ Secondly, we used a time dependent exposure definition that 


\begin{tabular}{|c|c|c|c|c|c|}
\hline \multirow[b]{2}{*}{ Exposure } & \multirow[b]{2}{*}{ Events } & \multirow[b]{2}{*}{ Person years } & \multirow[b]{2}{*}{ Incidence rate $(95 \% \mathrm{Cl}) *$} & \multicolumn{2}{|c|}{ Hazard ratio $(95 \% \mathrm{Cl})$} \\
\hline & & & & Crude & Adjustedt \\
\hline Use of other antidiabetic drugs & 159 & 460623 & 34.5 (29.4 to 40.3$)$ & 1.00 & 1.00 (reference) \\
\hline DPP-4 inhibitors & 49 & 91790 & $53.4(39.5$ to 70.6$)$ & 1.59 & 1.75 (1.22 to 2.49$)$ \\
\hline \multicolumn{6}{|c|}{$\begin{array}{l}\text { Cumulative duration of DPP- } 4 \text { inhibi- } \\
\text { tor use, years: }\end{array}$} \\
\hline$\leq 1$ & 16 & 36030 & $44.4(25.4$ to 72.1$)$ & 1.32 & $1.42(0.84$ to 2.41$)$ \\
\hline $1.1-2$ & 15 & 25491 & 58.8 (32.9 to 97.1$)$ & 1.70 & 1.91 (1.11 to 3.32$)$ \\
\hline $2.1-3$ & $\mathrm{~S} \ddagger$ & $\mathrm{S} \ddagger$ & $55.8(24.1$ to 110.0$)$ & 1.69 & $1.90(0.91$ to 3.96$)$ \\
\hline 3.1-4 & 7 & 8423 & 83.1 (33.4 to 171.2$)$ & 2.56 & $2.90(1.31$ to 6.41$)$ \\
\hline$>4$ & $S \ddagger$ & $S \ddagger$ & $39.9(8.2$ to 116.7$)$ & 1.28 & $1.45(0.44$ to 4.76$)$ \\
\hline \multicolumn{6}{|l|}{$\begin{array}{l}\text { Time since first DPP- } 4 \text { inhibitor } \\
\text { prescription, years: }\end{array}$} \\
\hline$\leq 2$ & 15 & 38608 & $38.9(21.7$ to 64.1$)$ & 1.12 & $1.23(0.72$ to 2.11$)$ \\
\hline $2.1-4$ & 24 & 32385 & $74.1(47.5$ to 110.3$)$ & 2.24 & 2.50 (1.57 to 3.99) \\
\hline$>4$ & 10 & 20797 & $48.1(23.1$ to 88.4$)$ & 1.56 & $1.75(0.86$ to 3.58$)$ \\
\hline \multicolumn{6}{|c|}{$\begin{array}{l}\text { DPP- } 4=\text { dipeptidyl peptidase- } 4 \text {. } \\
\text { *Per } 100000 \text { person years. } \\
\text { †Adjusted for age, sex, year of cohort entry, body mass index, alcohol related disorders (including alcoholism, alcoholic cirrhosis of liver, alcoholic hepatitis, } \\
\text { and hepatic failure), smoking status, haemoglobin } A_{1 c} \text {, microvascular (nephropathy, neuropathy, retinopathy) and macrovascular (myocardial infarction, } \\
\text { stroke, peripheral arteriopathy) complications of diabetes, duration of treated diabetes, antidiabetic drugs used before cohort entry, use of aspirin, non- } \\
\text { steroidal anti-inflammatory drugs, hormonal replacement therapy, oral contraceptives, other autoimmune conditions, total number of unique non-diabetic } \\
\text { drugs in year before cohort entry. } \\
\ddagger \text { Numbers }<5 \text { are not shown, as per confidentiality policies of Clinical Practice Research Datalink. }\end{array}$} \\
\hline
\end{tabular}

allowed patients to contribute both unexposed and exposed person time, thereby eliminating immortal time bias. ${ }^{33}$ Type 2 diabetes and inflammatory bowel disease have been shown to share inflammatory pathways, ${ }^{34}$ although large population based studies have not reported an association between these two diseases. ${ }^{35}$ Nevertheless, we rigorously assessed the effect of possible residual confounding in several analyses; these analyses yielded consistent findings. Moreover, the null association observed with insulin (a last line treatment of which the users are typically at an advanced disease stage) as a negative control provides reassurance on the internal validity of our findings. Finally, our results remained highly consistent across a variety of sensitivity analyses intended to overcome different sources of bias.

Analysis
Primary analysis
One year exposure lag period
Restriction to clinically supported events
Competing risk
Stratification on screening age:
«60 years
$\geq 60$ years
Stricter exposure definition
Hierarchical exposure reclassification
Exclusion of thiazolidinediones
Marginal structural model
Disease risk score
Multiple imputation

0.4

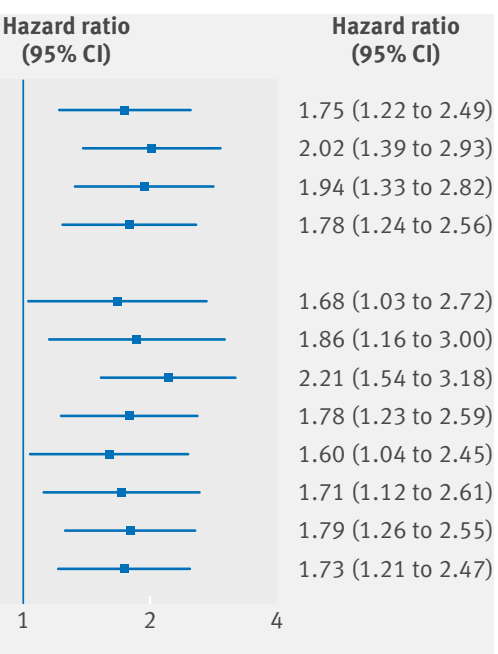

Fig 2 | Forest plot summarising results of primary analysis and sensitivity analyses, showing adjusted hazard ratios and $95 \% \mathrm{Cls}$ for association between use of dipeptidyl peptidase- 4 inhibitors and inflammatory bowel disease
Our study has a few limitations. As prescriptions in the CPRD are written by general practitioners and not specialists, some exposure misclassification is possible. In the UK, however, type 2 diabetes is managed almost entirely through primary care, ${ }^{36}$ so such misclassification is likely to have been minimal. Although inflammatory bowel disease has been shown to be well recorded in the CPRD, ${ }^{18-20}$ outcome misclassification is also possible. Reassuringly, we observed consistent findings in a sensitivity analysis using an algorithm based on clinically supporting events. Finally, as with all observational studies, residual confounding from unknown or unmeasured variables remains possible. However, on the basis of the rule out method, ${ }^{29}$ a hypothetical confounder would need to be strongly associated with both the exposure (odds ratio $>4.7$ ) and the outcome (relative risk $>5.0$ ) to move the point estimate towards the null (supplementary figure C). Whether such a hypothetical confounder exists beyond those considered in the analyses is unclear.

\section{Conclusions}

The results of this large population based cohort study indicate that the use of dipeptidyl peptidase- 4 inhibitors is associated with an overall $75 \%$ increase in the risk of inflammatory bowel disease in patients with type 2 diabetes. Although the absolute risk is low, physicians should be aware of this possible association and perhaps refrain from prescribing dipeptidyl peptidase-4 inhibitors for people at high risk (that is, those with a family history of disease or with known autoimmune conditions). Moreover, patients presenting with persistent gastrointestinal symptoms such as abdominal pain or diarrhoea should be closely monitored for worsening of symptoms. 


\begin{tabular}{|c|c|c|c|c|}
\hline Analysis & Events & Person years & Incidence rate $(95 \% \mathrm{CI})^{*}$ & Adjusted hazard ratio $(95 \% \mathrm{Cl}) \dagger \neq$ \\
\hline No use of insulin & 188 & 502896 & $37.4(32.2$ to 43.1$)$ & 1.00 (reference) \\
\hline Insulin & 18 & 44800 & $40.2(23.8$ to 63.5$)$ & $0.92(0.53$ to 1.58$)$ \\
\hline \multicolumn{5}{|c|}{ Head to head comparison } \\
\hline DPP-4 inhibitors & 40 & 77476 & $51.6(36.9$ to 70.3$)$ & $2.28(1.07$ to 4.85$)$ \\
\hline \multicolumn{5}{|c|}{$\begin{array}{l}\text { DPP-4=dipeptidyl peptidase-4. } \\
\text { *Per } 100000 \text { person years. } \\
\text { tInsulin negative control: adjusted for age, sex, year of cohort entry, body mass index, alcohol related disorders (including alcoholism, alcoholic cirrhosis of } \\
\text { liver, alcoholic hepatitis, and hepatic failure), smoking status, haemoglobin } \mathrm{A}_{1 \mathrm{c}} \text {, microvascular (nephropathy, neuropathy, retinopathy) and macrovascular } \\
\text { (myocardial infarction, stroke, peripheral arteriopathy) complications of diabetes, duration of treated diabetes, antidiabetic drugs used before cohort entry, } \\
\text { use of aspirin, non-steroidal anti-inflammatory drugs, hormonal replacement therapy, oral contraceptives, other autoimmune conditions, total number of } \\
\text { unique non-diabetic drugs in year before cohort entry. } \\
\text { †Head to head comparison: stratified on fifths of propensity score. }\end{array}$} \\
\hline
\end{tabular}

Contributors: All authors conceived and designed the study. LA acquired the data. DA, AD, HY, and LA did the statistical analyses. All authors analysed and interpreted the data. DA wrote the manuscript, and all authors critically revised it. All authors approved the final version of the manuscript and agree to be accountable for the accuracy of the work. LA supervised the study and is the guarantor. Funding: This study was funded by a foundation scheme grant from the Canadian Institutes of Health Research. The sponsor had no influence on design and conduct of the study; collection, management, analysis, and interpretation of the data; and preparation, review, or approval of the manuscript. $A D$ is the recipient of a research fellowship from the German Research Foundation (Deutsche Forschungsgemeinschaft, DFG). LA holds a ChercheurBoursier junior 2 award from the Fonds de Recherche du Québec Santé and is the recipient of a William Dawson scholar award from McGill University.

Competing interests: All authors have completed the ICMJE uniform disclosure form at www.icmje.org/coi_disclosure.pdf (available on request from the corresponding author) and declare: this study was funded by the Canadian Institutes of Health Research; no financial relationships with any organisations that might have an interest in the submitted work in the previous three years; no other relationships or activities that could appear to have influenced the submitted work.

Ethical approval: The study protocol was approved by the Independent Scientific Advisory Committee of the Clinical Practice Research Datalink (protocol number 17_165R) and by the Research Ethics Board of Jewish General Hospital, Montreal, Quebec, Canada.

Data sharing: No additional data available.

Transparency: The guarantor ( $L A)$ affirms that this manuscript is an honest, accurate, and transparent account of the study being reported; that no important aspects of the study have been omitted; and that any discrepancies from the study as planned (and, if relevant, registered) have been explained.

This is an Open Access article distributed in accordance with the Creative Commons Attribution Non Commercial (CC BY-NC 4.0) license, which permits others to distribute, remix, adapt, build upon this work non-commercially, and license their derivative works on different terms, provided the original work is properly cited and the use is noncommercial. See: http://creativecommons.org/licenses/by-nc/4.0/.

1 Christensen DH, Rungby J, Thomsen RW. Nationwide trends in glucose-lowering drug use, Denmark, 1999-2014. Clin Epidemiol 2016;8:381-7. 10.2147/CLEP.S113211

2 Thornberry NA, Gallwitz B. Mechanism of action of inhibitors of dipeptidyl-peptidase-4 (DPP-4). Best Pract Res Clin Endocrinol Metab 2009;23:479-86. 10.1016/j.beem.2009.03.004

3 Nauck MA, Vilsbøll T, Gallwitz B, Garber A, Madsbad S. Incretinbased therapies: viewpoints on the way to consensus. Diabetes Care 2009;32(Suppl 2):S223-31. 10.2337/dc09-S315

4 Son JW, Kim S. Dipeptidyl Peptidase 4 Inhibitors and the Risk of Cardiovascular Disease in Patients with Type 2 Diabetes: A Tale of Three Studies. Diabetes Metab / 2015;39:373-83. 10.4093/ dmj.2015.39.5.373

5 Chen X. Biochemical Properties of Recombinant Prolyl Dipeptidases DPP-IV and DPP8. In: Lendeckel U, Reinhold D, Bank U, eds. Dipeptidyl Aminopeptidases: Basic Science and Clinical Applications. Springer US, 2006: 27-3210.1007/0-38732824-6 3.
6 Ohnuma K, Hosono O, Dang NH, Morimoto C. Dipeptidyl peptidase in autoimmune pathophysiology. Adv Clin Chem 2011;53:51-84. 10.1016/B978-0-12-385855-9.00003-5

7 Klemann C, Wagner L, Stephan M, von Hörsten S. Cut to the chase: a review of CD26/dipeptidyl peptidase-4's (DPP4) entanglement in the immune system. Clin Exp Immunol 2016;185:1-21. 10.1111/ cei.12781

8 Yazbeck R, Howarth GS, Abbott CA. Dipeptidyl peptidase inhibitors, an emerging drug class for inflammatory disease? Trends Pharmacol Sci 2009;30:600-7. 10.1016/j.tips.2009.08.003

9 Yazbeck R, Howarth GS, Geier MS, Demuth HU, Abbott CA. Inhibiting dipeptidyl peptidase activity partially ameliorates colitis in mice. Front Biosci 2008;13:6850-8. 10.2741/3193

10 Mimura S, Ando T, Ishiguro K, et al. Dipeptidyl peptidase-4 inhibitor anagliptin facilitates restoration of dextran sulfate sodium-induced colitis. Scand J Gastroenterol 2013;48:1152-9. 10.3109/00365521.2013.832366

11 Magro DO, Kotze PG, Martinez CAR, et al. Changes in serum levels of lipopolysaccharides and CD26 in patients with Crohn's disease. Intest Res 2017;15:352-7. 10.5217/ir.2017.15.3.352

12 Moran GW, O’Neill C, Padfield P, McLaughlin JT. Dipeptidyl peptidase-4 expression is reduced in Crohn's disease. Regul Pept 2012;177:40-5. 10.1016/j.regpep.2012.04.006

13 Hildebrandt M, Rose M, Rüter J, Salama A, Mönnikes H, Klapp BF. Dipeptidyl peptidase IV (DP IV, CD26) in patients with inflammatory bowel disease. Scand I Gastroenterol 2001;36:1067-72. 10.1080/003655201750422675

14 Herrett E, Thomas SL, Schoonen WM, Smeeth L, Hall AJ. Validation and validity of diagnoses in the General Practice Research Database: a systematic review. Br J Clin Pharmacol 2010;69:4-14. 10.1111/j.1365-2125.2009.03537.x

15 Jick SS, Kaye JA, Vasilakis-Scaramozza C, et al. Validity of the general practice research database. Pharmacotherapy 2003;23:686-9. 10.1592/phco.23.5.686.32205

16 Lawrenson R, Williams T, Farmer R. Clinical information for research; the use of general practice databases. J Public Health Med 1999;21:299-304. 10.1093/pubmed/21.3.299

17 U.S. National Library of Medicine. RCD (Read codes) - synopsis. 2017. https://www.nlm.nih.gov/research/umls/sourcereleasedocs/ current/RCD/.

18 Lewis JD, Brensinger C, Bilker WB, Strom BL. Validity and completeness of the General Practice Research Database for studies of inflammatory bowel disease. Pharmacoepidemiol Drug Saf 2002;11:211-8. 10.1002/pds.698

19 García Rodríguez LA, González-Pérez A, Johansson S, Wallander MA. Risk factors for inflammatory bowel disease in the general population. Aliment Pharmacol Ther 2005;22:309-15. 10.1111/j.1365-2036.2005.02564.x

20 van Staa TP, Cooper C, Brusse LS, Leufkens H, Javaid MK, Arden NK Inflammatory bowel disease and the risk of fracture. Gastroenterology 2003;125:1591-7. 10.1053/j.gastro.2003.09.027

21 Louis E. When it is not inflammatory bowel disease: differential diagnosis. Curr Opin Gastroenterol 2015;31:283-9. 10.1097/ MOG.0000000000000183

22 Vavricka SR, Spigaglia SM, Rogler G, et al, Swiss IBD Cohort Study Group. Systematic evaluation of risk factors for diagnostic delay in inflammatory bowel disease. Inflamm Bowel Dis 2012;18:496-505. 10.1002/ibd.21719

23 Ruigómez A, García Rodríguez LA, Johansson S, Wallander MA. Is hormone replacement therapy associated with an increased risk of irritable bowel syndrome?Maturitas 2003;44:133-40. 10.1016/ S0378-5122(02)00321-3 
24 Schneeweiss S, Seeger JD, Maclure M, Wang PS, Avorn J, Glynn RJ. Performance of comorbidity scores to control for confounding in epidemiologic studies using claims data. Am Epidemiol 2001;154:854-64. 10.1093/aje/154.9.854

25 Suissa D, Brassard P, Smiechowski B, Suissa S. Number needed to treat is incorrect without proper time-related considerations. / Clin Epidemiol 2012;65:42-6. 10.1016/j.jclinepi.2011.04.009

26 Fine JP, Gray RJ. A proportional hazards model for the subdistribution of a competing risk. I Am Stat Assoc 1999;94:49650910.1080/01621459.1999.10474144.

27 Public Health England. NHS bowel cancer screening (BCSP) programme. 2017. https://www.gov.uk/topic/population-screeningprogrammes/bowel.

28 Kim SC, Schneeweiss S, Glynn RJ, Doherty M, Goldfine AB Solomon DH. Dipeptidyl peptidase-4 inhibitors in type 2 diabetes may reduce the risk of autoimmune diseases: a population-based cohort study. Ann Rheum Dis 2015;74:1968-75. 10.1136/ annrheumdis-2014-205216

29 Schneeweiss S. Sensitivity analysis and external adjustment for unmeasured confounders in epidemiologic database studies of therapeutics. Pharmacoepidemiol Drug Saf 2006;15:291-303. $10.1002 / p d s .1200$

30 Lipsitch M, Tchetgen Tchetgen E, Cohen T. Negative controls: a tool for detecting confounding and bias in observational studies. Epidemiology 2010;21:383-8. 10.1097/EDE.0b013e3181d61eeb
31 Zietek T, Rath E. Inflammation Meets Metabolic Disease: Gut Feeling Mediated by GLP-1. Front Immunol 2016;7:154. 10.3389/ fimmu.2016.00154

32 Ray WA. Evaluating medication effects outside of clinical trials: new-user designs. Am J Epidemiol 2003;158:915-20. 10.1093/aje/ kwg 231

33 Suissa S. Immortal time bias in pharmaco-epidemiology. Am J Epidemiol 2008;167:492-9. 10.1093/aje/kwm324

34 Jurjus A, Eid A, Al Kattar S, et al. Inflammatory bowel disease, colorectal cancer and type 2 diabetes mellitus: The links. BBA Clin 2015;5(Supplement C):16-24. 10.1016/j. bbacli.2015.11.002

35 Bähler C, Schoepfer AM, Vavricka SR, Brüngger B, Reich O. Chronic comorbidities associated with inflammatory bowel disease: prevalence and impact on healthcare costs in Switzerland. Eur / Gastroenterol Hepatol 2017;29:916-25. 10.1097| MEG.0000000000000891

36 Sharma M, Nazareth I, Petersen I. Trends in incidence, prevalence and prescribing in type 2 diabetes mellitus between 2000 and 2013 in primary care: a retrospective cohort study. BMJ Open 2016;6:e010210. 10.1136/ bmjopen-2015-010210

\section{Supplementary materials}

